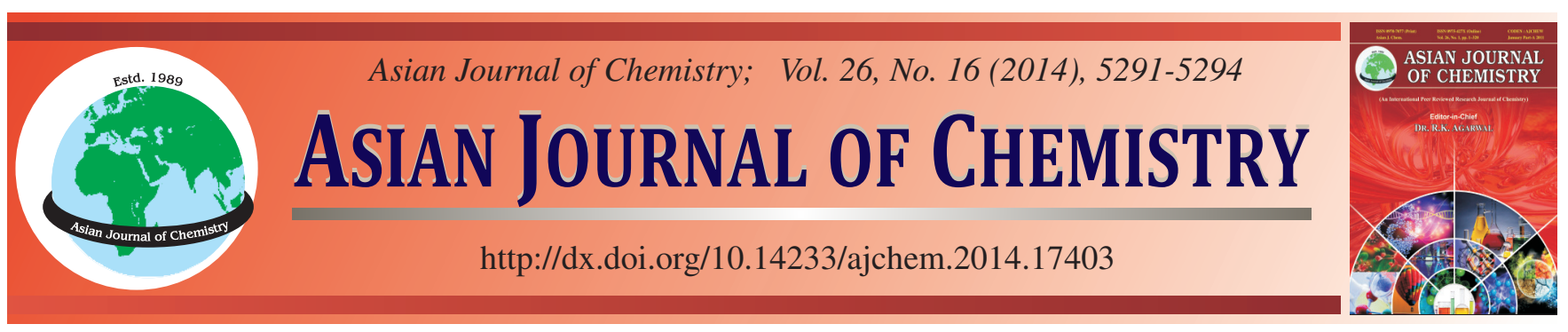

\title{
Synthesis and Antimicrobial Evaluation of 2-Aminobenzophenone Linked 1,4-Dihydropyridine Derivatives
}

\section{Pengying Li ${ }^{1}$, KetKi SAhore ${ }^{2}$, JiAnjun Liu ${ }^{1, *}$ and Rajesh K. Singh ${ }^{2, *}$}

${ }^{1}$ Forestry College, Northwest A and F University, Yangling 712100, P.R. China

${ }^{2}$ Department of Pharmaceutical Chemistry, Shivalik College of Pharmacy (Under Local Govt. Dept. Punjab) Nangal, Distt. Rupnagar-140126, Punjab, India

*Corresponding authors: E-mail: jianjun2013Liu@126.com; rksingh244@gmail.com

\begin{abstract}
A series of novel aminobenzophenone linked 1,4-dihydropyridines (1,4-DHPs) hybrids were synthesized by incorporating aminobenzophenone moiety on 1,4-dihydropyridines scaffold (5a-f) and characterized by IR, ${ }^{1} \mathrm{H}$ NMR and CHN elemental analysis. The synthesized compounds were tested for their in vitro antibacterial activity against the Gram-positive (Staphylococcus aureus) and Gramnegative (Escherichia coli) bacteria. Among all the synthesized compounds, compounds $\mathbf{5 b}, \mathbf{5} \mathbf{c}$ and $\mathbf{5 e}$ have shown the maximum activity in their group whereas compounds $\mathbf{5 a}, \mathbf{5 d}, \mathbf{5} \mathbf{f}$ have shown the minimum activity.
\end{abstract}

Keywords: 1,4-Dihydropyridine, 2-Aminobenzophenone, Pharmacophore, Antimicrobial activity.

\section{INTRODUCTION}

Nitrogen heterocyclic frameworks are prevalent in pharmaceuticals and biologically functional molecules. Among various nitrogen heterocyclics, 1,4-dihydropyridines $(1,4-$ DHP) scaffold is one of the most versatile pharmacophores since it has been found as the central core in many pharmaceuticals ${ }^{1-7}$ and so considered as most "privileged structure" in heterocyclic chemistry that, when appropriately substituted can exert potent and diverse pharmacological actions as shown in Fig. 1.

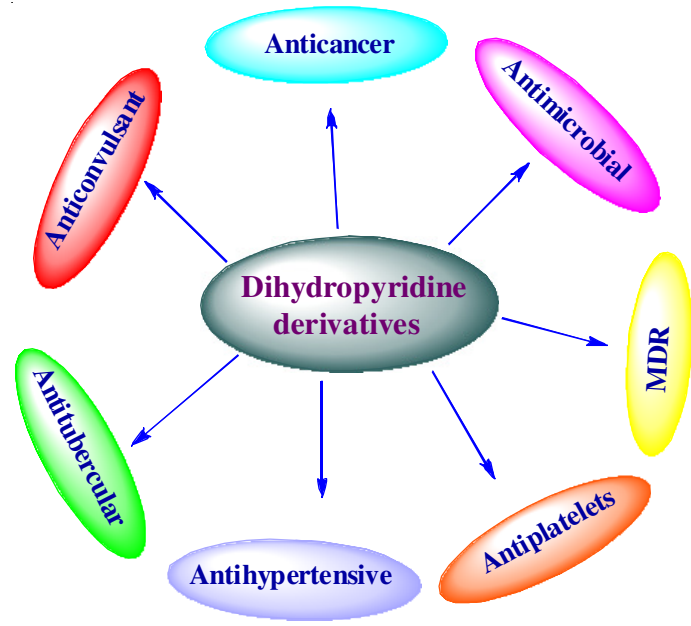

Fig. 1. Pharmacological activities exhibited by dihydropyridine derivatives
Recent studies have revealed that 1,4-DHPs exhibit potent antimicrobial activities ${ }^{8-13}$ and lots of works have been going on focusing 1,4-dihydropyridines synthesis as antibacterial chemotherapeutic regimen (Fig. 2).

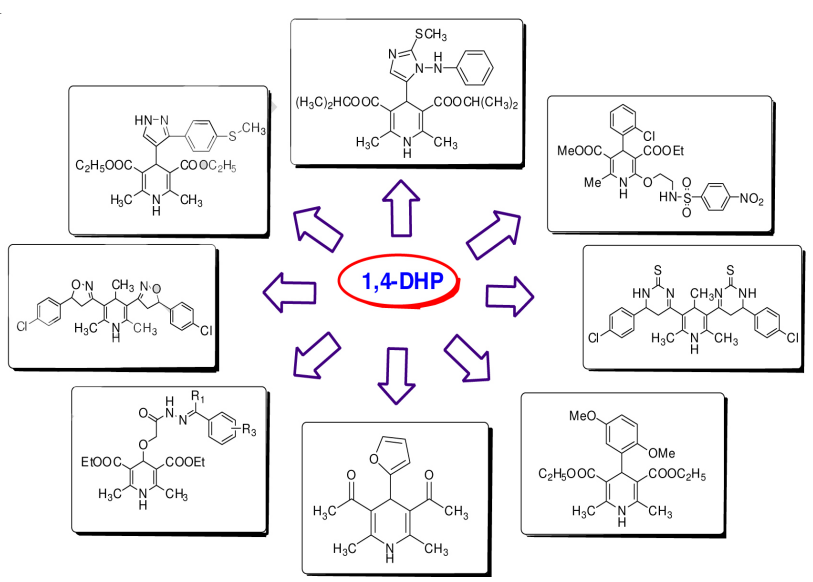

Fig. 2. Different derivatives of 1,4-dihydropyridine nucleus having good antimicrobial activities

2-Aminobenzophenone derivatives are important compounds in organic chemistry because of their application in heterocyclic synthesis and medicines ${ }^{14}$. Some heterocyclic compounds containing 2-aminobenzophenone moiety possess anti-inflammatory ${ }^{15}$, anti-tumor ${ }^{16}$, antimitotic ${ }^{17}$, anti-viral ${ }^{18}$ and skeletal muscle relaxant activities ${ }^{19}$. More recently, some 
research workers identified the antimicrobial potential of benzophenone nucleus and much research has been focused on the synthesis of its various derivatives for antibacterial and antifungal activities ${ }^{20,21}$.

The combination of two pharmacophores in a single molecule may enable synergistic interaction, where the efficacy of a single agent is enhances by the addition of a second compound. In view of these observations, it was decided to synthesize aminobenzophenone linked 1,4-dihydropyridines derivatives by incorporating aminobenzophenone pharmacophore on 1,4-dihydropyridines scaffold (Fig 3.) and screen them for their level of antimicrobial activity in the hope to obtained potent antibacterial agents.
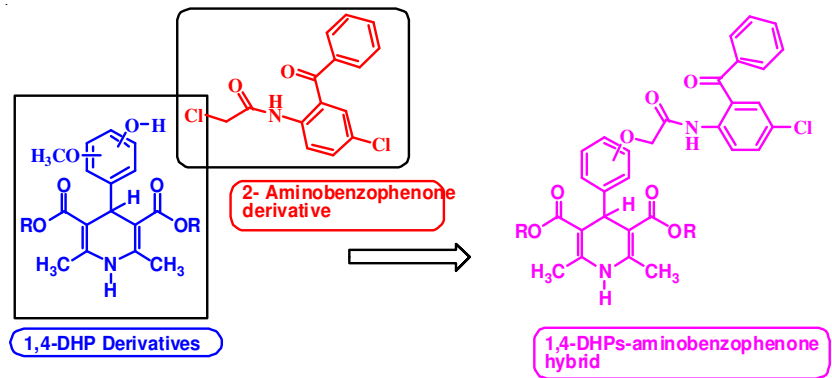

Fig. 3. Design of aminobenzophenone linked 1,4-dihydropyridine derivatives as possible antimicrobial agents

\section{EXPERIMENTAL}

The melting points were determined on Veego-programmable melting point apparatus (microprocessor based) and are uncorrected. Infrared (IR) spectra were obtained with on a Perkin Elmer 882 Spectrum and RXI, FT-IR model using a potassium bromide pellets $(\mathrm{KBr})$ in $\mathrm{cm}^{-1}$. ${ }^{1} \mathrm{H}$ NMR spectra were obtained using Brucker AC-400 F, $400 \mathrm{MHz}$ spectrometer and are reported in parts per million (ppm), downfield from tetramethylsilane (TMS) as internal standard. To monitor the reactions, as well as to established the identity and purity of reactants and products, thin layer chromatography was performed on microscopic slides $(2 \times 7.5 \mathrm{~cm})$, which were coated with silica gel $\mathrm{G}$ and activated at $110^{\circ} \mathrm{C}$ for $0.5 \mathrm{~h}$. The plates were developed by exposure to iodine vapours. All the chemicals used in the synthesis are of analytical grade. Anhydrous sodium sulphate was utilized as drying agents. All the solvents were dried and freshly distilled prior to use according to standard procedure. The starting compound 2-(chloroacetamido)-5-chlorobenzophenone and substituted 1,4-dihydroyridines were earlier synthesized in our lab by conventional as well as microwave irradiation technique 19,22,23. $^{2}$.

General method for the synthesis of dimethyl/diethyl4/3(substituted)-2-oxoethoxy)-4-methoxyphenyl)-2,6dimethyl-1, 4-dihydropyridine-3,5-dicarboxylate (5a-5f)

Method A: A mixture of 2-(chloroacetamido)-5-chlorobenzophenone ( $1.23 \mathrm{~g}, 4 \mathrm{mmol})$, potassium fluoride $(0.116 \mathrm{~g}$, $2 \mathrm{mmol})$ and dry powdered potassium carbonate $(0.7 \mathrm{~g}, 5$ $\mathrm{mmol})$ in dry DMF $(15 \mathrm{~mL})$ was stirred at room temperature for $1 \mathrm{~h}$. A solution of substituted 1,4-dihydropyridines (1 mmol) in dry DMF $(5 \mathrm{~mL})$ was added into the above mixture and it was stirred at room temperature for $24 \mathrm{~h}$. The reaction mixture was poured onto ice water $(100 \mathrm{~mL})$ and extracted with EtOAc $(3 \times 50 \mathrm{~mL})$. The organic extracts were combined, washed with ice water, dried over $\mathrm{Na}_{2} \mathrm{SO}_{4}$ and evaporated in vacuo to dryness. Residue was recrystallised from EtOH to get products (5a-f).

Method B: A mixture of substituted 1,4-dihydropyridines ( $2 \mathrm{mmol}$ ) and anhydrous potassium carbonate $(0.7 \mathrm{~g}, 5 \mathrm{mmol})$ in ethyl methyl ketone $(50 \mathrm{~mL})$ was refluxed for $2 \mathrm{~h}$ with stirring. To this, 2-(chloroacetamido)-5-chlorobenzophenone (1.23 $\mathrm{g}, 4 \mathrm{mmol})$ was added and the reaction mixture was refluxed for another $6 \mathrm{~h}$. The resulting slurry was filtered off and the solvent was recovered under reduced pressure to obtain oily residues which was recrystallized from ethanol to get products (5a-f).

Dimethyl 4-[4-\{2-((2-benzoyl-4-chlorophenyl)amino)2-oxoethoxy\}-3-methoxyphenyl]-2,6-dimethyl-1,4-dihydropyridine-3,5-dicarboxylate (5a): Yield $=\operatorname{method} \mathbf{A}: 71 \%$, method B $65 \%$, m.p. $112-115^{\circ} \mathrm{C} ; \mathrm{R}_{\mathrm{f}}=0.62$ [chloroform: methanol] [9:1], IR (KBr, $\left.v_{\max }, \mathrm{cm}^{-1}\right): 3350$ ( $\mathrm{Sec} \mathrm{N}-\mathrm{H}$ str. of DHP), 3088 (aromatic C-H str.), 2984 (C-H alkanes str.), 1681( $>\mathrm{C}=\mathrm{O}$ str. ester), 1488 ( $\mathrm{C}=\mathrm{C}$ aromatic ring), 1214 (C-O str.) and $750(\mathrm{C}-\mathrm{Cl}) .{ }^{1} \mathrm{H} \mathrm{NMR}\left(\mathrm{CDCl}_{3}\right) \delta(\mathrm{ppm}): 2.33(\mathrm{~s}, 6 \mathrm{H}$, $\left.2 \times \mathrm{CH}_{3}\right), 3.64\left(\mathrm{~s}, 2 \mathrm{H}, \mathrm{OCH}_{2}\right), 3.65\left(\mathrm{~s}, 6 \mathrm{H}, 2 \times \mathrm{COOCH}_{3}\right)$, 3.83 (s, 3H, - $\mathrm{OCH}_{3}$ ), 4.93 (s, 1H, -CH of DHP), 5.63 (s, 1H, $\mathrm{NH}$ of DHP) and 6.71-7.74 (m, 11H, ArH), 8.61 (s, 1H, NH). Anal. calcd. for $\mathrm{C}_{33} \mathrm{H}_{31} \mathrm{~N}_{2} \mathrm{O}_{8} \mathrm{Cl}: \mathrm{C} \%$ 64.02, $\mathrm{H} \% 5.05, \mathrm{~N} \%$ 4.53; Found: C \% 64.22, H \% 5.18, N \% 4.58

Dimethyl 4-[3-\{2-((2-benzoyl-4-chlorophenyl)amino)2-oxoethoxy\}phenyl]-2,6-dimethyl-1, 4-dihydropyridine3,5-dicarboxylate (5b): Yield = method A $68 \%$, method B $62 \%$, m.p. $163-165{ }^{\circ} \mathrm{C} ; \mathrm{R}_{\mathrm{f}}=0.68$ [chloroform:methanol] [9:1] IR (KBr, $v_{\max }, \mathrm{cm}^{-1}$ ): 3329 (N-H str.), 3091(ar C-H str.), 3000 (C-H str. alkanes), 1681 ( $>\mathrm{C}=\mathrm{O}$ str. amide), 1484 (C=C str. ar. ring), $1348(\mathrm{C}-\mathrm{N}), 1227$ (C-O str. ester) and $759(\mathrm{C}-\mathrm{Cl}) .{ }^{1} \mathrm{H}$ NMR (DMSO- $\left.d_{6}\right) \delta(\mathrm{ppm}): 2.27\left(\mathrm{~s}, 6 \mathrm{H}, 2 \times \mathrm{CH}_{3}\right), 3.58(\mathrm{~s}, 6 \mathrm{H}$, $\left.2 \times \mathrm{COOCH}_{3}\right), 3.94\left(\mathrm{~s}, 2 \mathrm{H}, \mathrm{OCH}_{2}\right), 4.63(\mathrm{~s}, 1 \mathrm{H},-\mathrm{CH}$ of DHP), 4.9 (s, 1H, NH of DHP), 6.89-8.06 (m, 12H, ArH), 10.6 (s, $1 \mathrm{H}, \mathrm{N} H$ ). Anal. calcd. for $\mathrm{C}_{32} \mathrm{H}_{29} \mathrm{~N}_{2} \mathrm{O}_{7} \mathrm{Cl}$ : $\mathrm{C} \% 65.25, \mathrm{H} \% 4.96$, N \% 4.76; Found: C \% 65.58, H \% 5.12, N \% 4.62

Diethyl 4-[4-\{2-((2-benzoyl-4-chlorophenyl)amino)-2oxoethoxy\}-3-methoxyphenyl]-2,6-dimethyl-1,4-dihydropyridine-3,5-dicarboxylate $(5 \mathbf{c})$ : Yield $=\operatorname{method} \mathbf{A} 67 \%$, method B $60 \%$, m.p. $128-130{ }^{\circ} \mathrm{C} ; \mathrm{R}_{\mathrm{f}}=0.85$ [chloroform: methanol] [9:1] IR (KBr, $\left.v_{\max }, \mathrm{cm}^{-1}\right): 3332$ (N-H str. of DHP), 3061(ar. C-H str.), 2976 (C-H str. alkanes), 1691 (> C=O str. ester), 1596 (C=C Ar), 1505 (N-H bend), 1212 (C-O str. ester) and $748(\mathrm{C}-\mathrm{Cl}) .{ }^{1} \mathrm{H}$ NMR $\left(\mathrm{CDCl}_{3}\right) \delta(\mathrm{ppm}): 1.22-1.25(\mathrm{~m}, 6 \mathrm{H}$, $\left.2 \times \mathrm{OCH}_{2} \mathrm{CH}_{3}\right), 2.32\left(\mathrm{~s}, 6 \mathrm{H}, 2 \times \mathrm{CH}_{3}\right), 3.83\left(\mathrm{~s}, 5 \mathrm{H},-\mathrm{OCH}_{3}+\right.$ $\left.\mathrm{OCH}_{2}\right), 4.07-4.10\left(\mathrm{q}, 4 \mathrm{H}, 2 \times \mathrm{OCH}_{2} \mathrm{CH}_{3}\right), 4.94(\mathrm{~s}, 1 \mathrm{H}, \mathrm{CH}$ of DHP), 5.54 (s, 1H, NH of DHP), 6.84-7.82 (m, 11H, ArH), 8.60 (s, $1 \mathrm{H}, \mathrm{NH}$ ). Anal. calcd. for $\mathrm{C}_{35} \mathrm{H}_{35} \mathrm{~N}_{2} \mathrm{O}_{8} \mathrm{Cl}: \mathrm{C} \% 64.96$, $\mathrm{H} \%$ 5.45, N \% 4.33; Found: C \% 65.24, H \% 5.70, N \% 4.45

Diethyl 4-[4-\{2-((2-benzoyl-4-chlorophenyl)amino)-2oxoethoxy\}phenyl]-2,6-dimethyl-1,4-dihydropyridine-3,5dicarboxylate (5d): Yield $=$ method A $74 \%$, method B 64 $\%$, m.p. 158-161 ${ }^{\circ} \mathrm{C} ; \mathrm{R}_{\mathrm{f}}=0.82$ [chloroform:methanol] [9:1] IR (KBr, $\left.v_{\max }, \mathrm{cm}^{-1}\right): 3347$ (N-H str.), 2984 (C-H str. alkanes), 1659 (> C=O str. amide), $1486(\mathrm{C}=\mathrm{C}$ str. ar. ring $), 1333(\mathrm{C}-\mathrm{N})$, 1228 (C-O str. ester) and $745(\mathrm{C}-\mathrm{Cl}) .{ }^{1} \mathrm{H}$ NMR (DMSO- $\left.d_{6}\right)$ 
$\delta$ (ppm): 1.05-1.09 (t, 6H, $\left.2 \times \mathrm{OCH}_{2} \mathrm{CH}_{3}\right), 2.18(\mathrm{~s}, 6 \mathrm{H}, 2 \times$ $\left.\mathrm{CH}_{3}\right), 3.35\left(\mathrm{~s}, 2 \mathrm{H}, \mathrm{OCH}_{2}\right), 3.89-3.98\left(\mathrm{~m}, 4 \mathrm{H}, 2 \times \mathrm{OCH}_{2} \mathrm{CH}_{3}\right)$, 4.68 (s, 1H, CH of DHP), 6.49-7.48 (m, 12H, ArH), 8.29 (s, $1 \mathrm{H}, \mathrm{NH})$. Anal. calcd. for $\mathrm{C}_{34} \mathrm{H}_{33} \mathrm{~N}_{2} \mathrm{O}_{7} \mathrm{Cl}: \mathrm{C} \%$ 66.18, $\mathrm{H} \%$ 5.39, N\% 4.54; Found: C\% 66.58, H\% 5.66, N\% 4.58

Diethyl 4-[3-\{2-((2-benzoyl-4-chlorophenyl)amino)-2oxoethoxy\}-4-methoxyphenyl]-2,6-dimethyl-1,4-dihydropyridine-3,5-dicarboxylate (5e): Yield $=\operatorname{method} \mathbf{A} 72 \%$, method B $65 \%$, m.p. $198-200{ }^{\circ} \mathrm{C} ; \mathrm{R}_{\mathrm{f}}=0.65$ [chloroform: methanol] [9:1] IR (KBr, $\left.v_{\max }, \mathrm{cm}^{-1}\right): 3356(\mathrm{~N}-\mathrm{H}$ str. of DHP), 3090 (aromatic C-H str.), 2958 (C-H str. alkanes), 1696 (ester $\mathrm{C}=\mathrm{O}$ ), 1486 (aromatic $\mathrm{C}=\mathrm{C}), 1214$ (ether $\mathrm{C}-\mathrm{O}), 780(\mathrm{C}-\mathrm{Cl})$. ${ }^{1} \mathrm{H} \mathrm{NMR}\left(\mathrm{CDCl}_{3}\right) \delta(\mathrm{ppm}): 1.23-1.28\left(\mathrm{~m}, 6 \mathrm{H}, 2 \times \mathrm{OCH}_{2} \mathrm{CH}_{3}\right)$, $2.27\left(\mathrm{~s}, 3 \mathrm{H}, \mathrm{CH}_{3}\right), 2.88\left(\mathrm{~s}, 3 \mathrm{H}, \mathrm{CH}_{3}\right), 3.77-3.82\left(\mathrm{~s}, 5 \mathrm{H}, \mathrm{OCH}_{3}\right.$ $\left.+\mathrm{OCH}_{2}\right), 4.04-4.1$ (q, $\left.4 \mathrm{H}, \mathrm{OCH}_{2} \mathrm{CH}_{3}\right), 4.58(\mathrm{~s}, 1 \mathrm{H}, \mathrm{CH}$ of DHP), 4.89 (s, 1H, NH of DHP),6.66-7.72 (m, 11H, ArH), 8.62 (s, $1 \mathrm{H}, \mathrm{NH}$ ). Anal. calcd. for $\mathrm{C}_{35} \mathrm{H}_{35} \mathrm{~N}_{2} \mathrm{O}_{8} \mathrm{Cl}: \mathrm{C} \%$ 64.96, $\mathrm{H} \%$ 5.45, N\% 4.33; Found: C \% 63.87, H\% 5.89, N\% 4.68.

Dimethyl 4-[3-\{2-((2-benzoyl-4-chlorophenyl)amino)2-oxoethoxy\}-4-methoxyphenyl]-2,6-dimethyl-1,4-dihydro-

Step 1<smiles>CC(=O)CC(=O)O</smiles>

(1)

$\mathrm{R}=\mathrm{CH}_{3}$ or $\mathrm{CH}_{2} \mathrm{CH}_{3}$<smiles>[R2]c1ccc(C=O)cc1</smiles>

(2)
The reaction pathway used for the synthesis of title compounds have been shown in Scheme-I. The final compounds were prepared by two methods (methods A and B) to optimize the yield. Method A includes the stirring at room temperature of 2-(chloroacetamido)-5-chlorobenzophenone (4) and substituted 1,4-dihydropyridines (3a-f) in the presence of potassium carbonate and sodium iodide in a minimum quantity of DMF and by refluxing 2-(chloroacetamido)-5-chlorobenzophenone<smiles>[R2]C(=O)C1=C(C)NC(C)=C(C(=O)O)C1(C(=O)O[Na])c1ccc([R])c([R])c1</smiles>

method B $62 \%$, m.p. $165-167{ }^{\circ} \mathrm{C} ; \mathrm{R}_{\mathrm{f}}=0.63$ [chloroform: methanol] [9:1] IR ( $\left.\mathrm{KBr}, \mathrm{v}_{\max }, \mathrm{cm}^{-1}\right): 3345$ (N-H str. of DHP), 2982 (C-H str. alkanes), 1673 (ester $\mathrm{C}=\mathrm{O}), 1487$ ( $\mathrm{Ar} \mathrm{C}=\mathrm{C})$, 1228 (ether $\mathrm{C}-\mathrm{O}), 759(\mathrm{C}-\mathrm{Cl}) .{ }^{1} \mathrm{H}$ NMR $\left(\mathrm{CDCl}_{3}\right) \delta(\mathrm{ppm})$ : $2.27\left(\mathrm{~s}, 6 \mathrm{H}, 2 \times \mathrm{CH}_{3}\right), 3.54\left(\mathrm{~s}, 2 \mathrm{H}, \mathrm{OCH}_{2}\right), 3.61(\mathrm{~s}, 6 \mathrm{H}, 2 \times$ $\left.\mathrm{COCH}_{3}\right), 3.81\left(\mathrm{~s}, 3 \mathrm{H},-\mathrm{OCH}_{3}\right), 4.58(\mathrm{~s}, 1 \mathrm{H}, \mathrm{CH}$ of DHP), $70(\mathrm{~s}, 1 \mathrm{H}, \mathrm{NH}$ of DHP), 6.68-7.75 (m, 11H, $\operatorname{Ar} H), 8.62$ (s, $1 \mathrm{H}, \mathrm{N} H$ ) Anal. calcd. for $\mathrm{C}_{33} \mathrm{H}_{31} \mathrm{~N}_{2} \mathrm{O}_{8} \mathrm{Cl}$ : $\mathrm{C} \%$ 64.02, $\mathrm{H} \%$ 5.05, N\% 4.53; Found: C\% 64.22, H\% 5.16, N\% 4.60

\section{RESULTS AND DISCUSSION}

(3)

$\begin{array}{llcl} & \mathbf{R}_{1} & \mathbf{R}_{\mathbf{2}} & \mathbf{R} \\ \text { 3a } & \mathrm{OH} & \mathrm{OCH}_{3} & \mathrm{CH}_{3} \\ \text { 3b } & \mathrm{H} & \mathrm{OH} & \mathrm{CH}_{3} \\ \text { 3c } & \mathrm{OH} & \mathrm{OCH}_{3} & \mathrm{C}_{2} \mathrm{H}_{5} \\ \text { 3d } & \mathrm{OH} & \mathrm{H} & \mathrm{C}_{2} \mathrm{H}_{5} \\ \text { 3e } & \mathrm{OCH}_{3} & \mathrm{OH} & \mathrm{C}_{2} \mathrm{H}_{5} \\ \text { 3f } & \mathrm{OCH}_{3} & \mathrm{OH} & \mathrm{CH}_{3}\end{array}$<smiles>[R2]c1ccc(C2(C(=O)O)C(C(=O)O)=C(C)NC(C)=C2C(=O)O)cc1</smiles>

(3af)<smiles>O=C(CCl)Nc1ccc(Cl)cc1C(=O)c1ccccc1</smiles>

(4)<smiles>[X]c1ccc(C2(C(=O)O)C(C(=O)O)=C(C)NC(C)=C2C(=O)O)cc1[X]</smiles>

(5 a-f)

\begin{tabular}{|c|c|c|c|c|c|c|}
\hline & $5 a$ & $5 b$ & $5 \mathrm{c}$ & $5 d$ & $5 e$ & $5 f$ \\
\hline $\mathrm{X}$ & & $\mathrm{H}$ & & & $\mathrm{OCH}_{3}$ & $\mathrm{OCH}_{3}$ \\
\hline Y & $\mathrm{OCH}_{3}$ & & $\mathrm{OCH}$ & $\mathrm{H}$ & & \\
\hline $\mathrm{R}$ & $\mathrm{CH}_{3}$ & $\mathrm{CH}_{3}$ & $\mathrm{C}_{2} \mathrm{H}_{3}$ & $\mathrm{C}_{2} \mathrm{H}_{5}$ & $\mathrm{C}_{2} \mathrm{H}_{5}$ & $\mathrm{CH}_{3}$ \\
\hline
\end{tabular}

Reagents \& conditions: (i) Ammonium acetate, $\mathrm{EtOH}$, reflux for $24 \mathrm{~h}$ (iia) Method A: $\mathrm{K}_{2} \mathrm{CO}_{3}, \mathrm{KI}$ and DMF, stirring at RT for $24 \mathrm{~h}$ (iib) Method B: $\mathrm{K}_{2} \mathrm{CO}_{3}$, ethyl methyl ketone, reflux with stirring for $6 \mathrm{~h}$. 


\begin{tabular}{|c|c|c|c|c|c|c|c|c|}
\hline \multirow{4}{*}{ Compounds } & \multicolumn{7}{|c|}{$\begin{array}{l}\text { TABLE-1 } \\
\text { ZONE OF INHIBITION (IN mm) OF Staphylococcus aureus AND Escherichia coli } \\
\text { OF TEST COMPOUNDS AT A CONCENTRATION OF 50, 100, 150 AND } 200 \mu \mathrm{G} / \mathrm{ML}\end{array}$} & \\
\hline & \multicolumn{8}{|c|}{ Zone of inhibition (mm) } \\
\hline & \multicolumn{4}{|c|}{ Staphylococcus aureus } & \multicolumn{4}{|c|}{ Escherichia coli } \\
\hline & $50(\mu \mathrm{g} / \mathrm{mL})$ & $100(\mu \mathrm{g} / \mathrm{mL})$ & $150(\mu \mathrm{g} / \mathrm{mL})$ & $200(\mu \mathrm{g} / \mathrm{mL})$ & $50(\mu \mathrm{g} / \mathrm{mL})$ & $100(\mu \mathrm{g} / \mathrm{mL})$ & $150(\mu \mathrm{g} / \mathrm{mL})$ & $200(\mu \mathrm{g} / \mathrm{mL})$ \\
\hline Amoxicillin & 12 & 13 & 17 & 18 & 10 & 12 & 15 & 18 \\
\hline $5 \mathbf{a}$ & 2 & 4 & 7 & 6 & 2 & 2 & 8 & 6 \\
\hline $5 \mathbf{b}$ & 3 & 5 & 8 & 8 & 3 & 4 & 9 & 8 \\
\hline $5 c$ & 2 & 6 & 9 & 7 & 2 & 2 & 8 & 6 \\
\hline $5 d$ & 2 & 4 & 8 & 6 & 2 & 3 & 9 & 5 \\
\hline $5 e$ & 2 & 5 & 10 & 7 & 2 & 3 & 11 & 7 \\
\hline $5 f$ & 2 & 3 & 7 & 6 & 2 & 4 & 6 & 6 \\
\hline
\end{tabular}

(4) and (3a-f) in ethyl methyl ketone as solvent in the presence of potassium carbonate as catalyst by method B. It was observed that from both methods, method $\mathbf{A}$ was found to give the better yield. The structures of the synthesized compounds were determined by IR, NMR and CHN elemental studies.

Antibacterial activity of synthesized compounds was tested by Agar plate diffusion method ${ }^{24}$ against two bacterial strains: Staphylococcus aureus (Gram+ve) (ATCC-29737) and Escherichia coli (Gram-ve) (ATCC-8739) procured from IMTECH, Chandigarh. After the setting of media, $2 \mathrm{~mL}$ of bacterial culture was poured in Petri dishes and sprayed over agar media. Different concentrations of test compound (50, 100,150 and $200 \mu \mathrm{g} / \mathrm{mL}$ ) were placed in each hole of the Petri dish. The plates were incubated at $37{ }^{\circ} \mathrm{C}$ for $24 \mathrm{~h}$ for bacteria strains. The diameter of zones of inhibition formed around the disc was measured after $24 \mathrm{~h}$ for the bacteria plates. Amoxicillin was used as standard or positive control while DMSO was used as a solvent and as negative controls. Although none of the synthesized compounds have shown to have good antibacterial activity. However (compounds $\mathbf{5 b}, \mathbf{5 c}, \mathbf{5 e}$ ) have shown the maximum activity in their group whereas (compounds $\mathbf{5 a}, \mathbf{5 d}, \mathbf{5 f}$ ) have shown the minimum activity (Table-1).

\section{Conclusion}

A series of 2-aminobenzophenone linked 1,4-dihydropyridine derivatives were synthesized, characterized and finally evaluated for their antibacterial activity but none of the compounds have exhibited significant antibacterial activity. The study revealed that 1,4-dihydropyridines nucleus is a rich source of exploitation. Hence further modification of 1,4dihydropyridines and 2-aminobenzophenone moiety by substitution of different groups at different positions may provide more potent antibacterial agents in future.

\section{ACKNOWLEDGEMENTS}

The authors are thankful to Principal and College Managing Committee, Shivalik College of Pharmacy, Nangal, India for providing the necessary facilities to carry out the research work. Thanks are also due to Panjab University, Chandigarh, India for cooperation in getting the spectral data and $\mathrm{CHN}$ elemental studies.

\section{REFERENCES}

1. R.R. Poondra, R.V. Nallamelli, C.L.T. Meda, B.N.V. Srinivas, A. Grover, J. Muttabathula, S.R. Voleti, B. Sridhar, M. Pal and K.V.L. Parsa, Bioorg. Med. Chem. Lett., 23, 1104 (2013).

2. R. Boer and V. Gekeler, Drugs Future, 20, 499 (1995).

3. V. M. Briukhanov, Exp. Clin. Pharmacol., 57, 47 (1994).

4. S. Gullapalli and P. Ramarao, Neuropharmacology, 42, 467 (2002).

5. S. Bahekar and D. Shinde, Acta Pharm., 52, 281 (2002).

6. R. León, C. Ríos, J. Marco-Contelles, O. Huertas, X. Barril, F.J. Luque, M.G. López, A.G. García and M. Villarroya, Bioorg. Med. Chem., 16, 7759 (2008).

7. J. Marco-Contelles, R. León, C. de los Ríos, A. Samadi, M. Bartolini, V. Andrisano, O. Huertas, X. Barril, F.J. Luque, M.I. Rodríguez-Franco, B. López, M.G. López, A.G. García, M. do Carmo Carreiras and M. Villarroya, J. Med. Chem., 52, 2724 (2009).

8. S. Ulloora, R. Shabaraya, R. Ranganathan and A.V. Adhikari, Eur. J. Med. Chem., 70, 341 (2013).

9. G.A. Wachter, M.C. Davis, A.R. Martin and S.G. Franzblau, J. Med. Chem., 41, 2436 (1998).

10. V.L.R. Sana, V.R. Katla, S. Chennamsetty, A. Shaik and N.R. Chamarthi, Der Pharm. Sinica, 4, 10 (2013).

11. A.M. Vijesh, A.M. Isloor, S.K. Peethambar, K.N. Shivananda, T. Arulmoli and N.A. Isloor, Eur. J. Med. Chem., 46, 5591 (2011).

12. Y.L.N. Murthy, A. Rajack, M.T. Ramji, J. Jesonbabu, C. Praveen and K.A. Lakshmi, Bioorg. Med. Chem. Lett., 22, 6016 (2012).

13. V. Niraimathi, A.J. Suresh, S. Latha and S.C. Bala, Int. J. Pharm. Pharm. Sci., 4, 212 (2012).

14. D.A. Walsh, Synthesis, 677 (1980).

15. E.R. Ottosen, M.D. Sørensen, F. Björkling, T. Skak-Nielsen, M.S. Fjording, H. Aaes and L. Binderup, J. Med. Chem., 46, 5651 (2003).

16. S. Cortez-Maya, E. Cortes, S. Hernández-Ortega, T. Ramirez Apan and M. Martínez-García, Synth. Commun., 42, 46 (2012).

17. J.P. Liou, C.W. Chang, J.S. Song, Y.N. Yang, C.F. Yeh, H.Y. Tseng, Y.K. Lo, Y.L. Chang, C.M. Chang and H.P. Hsieh, J. Med. Chem., 45, 2556 (2002).

18. P. Cheng, Q. Zhang, Y-B. Ma, Z-Y. Jiang, X.M. Zhang, F.X. Zhang and J.J. Chen, Bioorg. Med. Chem. Lett., 18, 3787 (2008).

19. R.K. Singh, S. Devi and D.N. Prasad, Arab. J. Chem., doi:10.1016/ i.arabjc. 2011.11.013.

20. L. Sun, J. Wu, M. Luo, X. Wang, M. Pan, Z. Gou and D. Sun, Molecules, 16, 9739 (2011).

21. A. Sakunpak and P. Panichayupakaranant, Food Chem., 130, 826 (2012).

22. P. Kaur, H. Sharma, R. Rana, D.N. Prasad and R.K. Singh, Asian J. Chem., 24, 5649 (2012).

23. P. Kaur and R.K. Singh, Chem. Sci. Trans, 2(S1), S295 (2013).

24. Indian Pharmacopoeia, Government of India, Ministry of Health and Welfare, Published by Controller of publication, New Delhi, II, A105 (1996). 